\title{
A priori and a posteriori error analysis for numerical homogenization: a unified framework
}

\author{
Assyr Abdulle \\ Section of Mathematics, Swiss Federal Institute of Technology \\ 1015 Lausanne, Switzerland \\ assyr.abdulle@epfl.ch
}

\begin{abstract}
Numerical methods coupling macroscopic and microscopic solvers for the efficient solution of partial differential equations with multiple scales have attracted much attention these last few years. Efficient implementations of such methods need a thorough understanding of the subtle interplay between the macroscopic scale (often the scale of interest) and the microscopic scale taking into account the fine-scale behavior of the problem of interest. This paper is concerned with the error analysis of a class of numerical homogenization methods, the so-called heterogeneous multiscale finite element methods. We discuss recent results obtained for the a priori and the a posteriori error analysis of these numerical methods and give a general framework to perform such analyses.
\end{abstract}

\section{Introduction}

The modeling of problems in engineering and the sciences taking into account physical processes at different scales is nowadays common (e.g. composite materials whose effective behavior depend on its microscopic constituents, flow in heterogeneous porous media, multiscale fracture modeling, etc.). In turn new numerical techniques have been developed for the solution of such problems [15]. In order to be efficient and robust, these numerical techniques must take into account discretization parameters at various scales. It is thus crucial to understand how the error propagates across scales, to know the asymptotic behavior of numerical approximations (in dependence of the various discretization parameters) and to quantify the quality of a computed numerical solution.

In this paper we discuss finite element methods (FEMs) for so-called homogenization problems, important for many applications [33]. Various numerical methods based on FEMs have been developed these last 
few years for homogenization problems and we refer to [7, Sec. 1] for an overview. We focus here on the finite element heterogeneous multiscale method (FE-HMM). This method is built in the framework of the heterogeneous multiscale method (HMM) introduced in [23], coupling a macroscale method with a microscale method able to recover the parameters of the effective problem on the fly.

First analysis of the FE-HMM was given in [2] and [24]. Nonlinear and stochastic problems were discussed and partially analyzed in [24] while robust convergence rates (i.e. independent of the small scale size $\varepsilon)$ were obtained in [2]. The aforementioned works were concerned with semi-discrete error analyses, i.e., analyses assuming an exact solution of micro problems involved in the numerical method. Such an approach is commonly taken for the analysis of multiscale numerical methods [25] (and the references therein), despite its limitation (the complexity and accuracy of micro-macro numerical methods depends on the interplay of micro and macro numerical discretization errors). Fully discrete error analysis for the HMM was first obtained [1], where sharp micro and macro convergence rates were obtained. In a series of papers, this analysis was extended to elastic problem [4] and to advection-diffusion problems [3]. Coupling between different types of solvers were analyzed in [5] (FEM and spectral method), and in [8] (discontinuous Galerkin method).

While a large body of literature concerning a priori error analysis for multiscale problems is nowadays available, a posteriori error analysis for such methods has only rarely been addressed. For the FE-HMM, a first attempt of such a posteriori error analysis was given in [32]. Relying on a reformulation of the numerical method in the two-scale framework [30], a posteriori error estimates were obtained in a two-scale norm. First a posteriori error estimates in the energy norm and for the physical variables were obtained in [10],[11]. The methodology used in these latter papers applies to more general oscillating tensors, sampling domains, micro-boundary conditions than the analysis given in [32].

In this paper we give a general framework allowing for both a priori and a posteriori error analysis for the FE-HMM. Recent results combined in a unified framework are discussed.

The paper is organized as follows:

Section 2. Model problem and homogenization

Section 3. Coupling macro and micro FE methods: the FE-HMM

Section 4. Preliminary results

Section 5. A priori error analysis

Section 6. A posteriori error analysis 
Notations. In this paper, $C, \tilde{C}>0$ denote a generic constant, independent of $\varepsilon$, whose value can change at any occurrence but which depends only on the quantities which are indicated explicitly. We use the usual Sobolev space $W^{s, p}(\Omega)$. For $p=2$ we use the notation $H^{s}(\Omega)$ and $H_{0}^{1}(\Omega)$, and denote by $W_{p e r}^{1}(Y)=\left\{v \in H_{p e r}^{1}(Y) ; \int_{Y} v d x=0\right\}$, where $H_{p e r}^{s}(Y)$ is defined as the closure of $\mathcal{C}_{\text {per }}^{\infty}(Y)$ (the subset of $\mathcal{C}^{\infty}\left(\mathbb{R}^{d}\right)$ of periodic functions in the unit cube $\left.Y=(0,1)^{d}\right)$ for the $H^{s}$ norm. We also use the usual norms on the $W^{s, p}(\Omega)$ Sobolev spaces (e.g. $\|u\|_{L^{p}(\Omega)}=\left(\int_{\Omega}|u(x)|^{p} d x\right)^{1 / p}$ for $L^{p}(\Omega)$ spaces $\left.1 \leq p<\infty\right)$ and refer the reader to standard textbooks for their definition. For a matrix $A \in \mathbb{R}^{d \times d}$ with entries $a_{i j}$, we denote its Frobenius norm by $\|A\|_{F}:=\sqrt{\operatorname{trace}\left(A^{T} A\right)}=\sqrt{\sum_{i j} a_{i j}^{2}}$.

\section{Model problem and homogenization}

We recall here the homogenization problem that we consider in this paper. Let $\Omega \subset \mathbb{R}^{d}(d=1,2,3)$ be a convex polygonal domain and consider the elliptic problem

$$
-\nabla \cdot\left(a^{\varepsilon} \nabla u_{\varepsilon}\right)=f \text { in } \Omega, \quad u_{\varepsilon}=0 \text { on } \partial \Omega,
$$

where the family of tensors $a^{\varepsilon}(x) \in\left(L^{\infty}(\Omega)\right)^{d \times d}$ (indexed by $\varepsilon$ ) are assumed to be symmetric and uniformly elliptic and bounded, i.e.,

$$
\exists \lambda, \Lambda>0 \text { such that } \lambda|\xi|^{2} \leq a^{\varepsilon}(x) \xi \cdot \xi \leq \Lambda|\xi|^{2}, \forall \xi \in \mathbb{R}^{d} \text { and } \forall \varepsilon,
$$

where $\varepsilon$ is a microscopic scale that characterizes the multiscale nature of the tensor $a^{\varepsilon}(x)$. We also assume that $f \in L^{2}(\Omega)\left(f \in H^{-1}(\Omega)\right.$ would also be possible). Owing to the uniform ellipticity and boundedness of the tensor $a^{\varepsilon}$, we obtain, thanks to the Lax-Milgram theorem, a family of solutions $\left\{u_{\varepsilon}\right\}$ which are bounded in $H_{0}^{1}(\Omega)$. Without any further assumption on the heterogeneities of the tensor $a^{\varepsilon}(x)$ one can show (using $G$ or $H$ convergence [21],[29]) that there exists a symmetric tensor $a^{0}(x)$ and a subsequence of $\left\{u_{\varepsilon}\right\}$ which weakly converges in $H_{0}^{1}(\Omega)$ to an element $u_{0} \in H_{0}^{1}(\Omega)$. The so-called homogenized or upscaled function $u_{0}$ can be shown to satisfy a homogenized equation which reads

$$
-\nabla \cdot\left(a^{0} \nabla u_{0}\right)=f \text { in } \Omega, \quad u_{0}=0 \text { on } \partial \Omega .
$$

The homogenized tensor $a^{0}(x)$ can be shown to be symmetric and to satisfy $\lambda|\xi|^{2} \leq a^{0}(x) \xi \cdot \xi \leq \Lambda|\xi|^{2}, \forall \xi \in \mathbb{R}^{d}$. As a consequence, the homogenized problem (2.3) has a unique solution. If $a^{\varepsilon}(x)$ has a more specific structure, for example if $a^{\varepsilon}(x)=a(x, x / \varepsilon)$ and is periodic in its second argument, then classical result show that whole sequence $\left\{u_{\varepsilon}\right\}$ weakly converges to an element $u_{0} \in H_{0}^{1}(\Omega)$ and the homogenized tensor 
$a^{0}(x)$ at $x \in \Omega$ can be characterized by an average (integral) of solutions of $d$ boundary value problems, the so-called "cell problems" (see for example [26],[19]). Notice that even in this fortunate case, one still has in general an infinite number of cell problems to solve.

The most obvious numerical strategy to compute a numerical homogenized solution consists of the following steps:

1. pre-processing: compute the homogenized tensor at predefined sampling domains around sampling points $x_{i} \in \Omega, i=1, \ldots, p$, by solving for each $x_{i} d$ boundary value problems involving the small scale tensor $a^{\varepsilon}$ (the cell problems);

2. compute a homogenized solution on a macro FE mesh using the interpolated precomputed homogenized data as input tensor.

Although straightforward, this strategy has several drawbacks:

1. the sequential strategy does not allow for a natural way to derive a priori or a posteriori error control of the whole procedure (depending on the accuracy of the numerically pre-computed homogenized tensors);

2. the sequential algorithm separates the computation of the homogenized parameters (pre-processing) from the computation of the homogenized solution, while the simultaneous coupling between scales (described below) allows for a variety of (potentially more efficient) coupling (also for nonperiodic, nonlinear problems);

3. the algorithm does not allow, in a straightforward way, to switch locally to the fine scale solver in regions of the computational domain where the homogenized solution does not give an accurate description of the physics.

The macro-to-micro framework introduced below is based on a simultaneous coupling of the spatial scales. This framework gives a methodology to extend the numerical methods for more general problems (e.g. non-linear, time-dependent, wave problems) and more general numerical methods (coupling FEM with spectral or discontinuous Galerkin methods). As an example, highlighting the difference between the standard sequential strategy and the macro-to-micro method proposed below consider discontinuous Galerkin methods. As for discontinuous Galerkin methods fluxes perpendicular to the edges of the (macro) elements need to be defined, one would be tempted to compute cell problems on edges of the triangulation with the "sequential homogenization numerical method". An efficient coupling with a macro-to-micro method allows to avoid these additional cell problems since macro fluxes can be recovered from the available micro solutions computed in sampling domains and already needed for continuous FEM. 
A priori and a posteriori analysis for numerical homogenization 5

\section{Coupling macro and micro FE methods: the FE-HMM}

We describe in this section a numerical method for homogenization problems, the so-called finite element heterogeneous multiscale method (FE-HMM).

Macro finite element space. Let $\mathcal{T}_{H}$ be a (macro) partition of $\Omega$ in simplicial or quadrilateral elements $K$ of diameter $H_{K}$ and denote $H=\max _{K \in \mathcal{T}_{H}} H_{K}$. By macro partition we mean that $H$ can be much larger than $\varepsilon$ and that $H<\varepsilon$ is not necessary for convergence. We assume that the triangulation is conformal and shape regular (see [18]). For this partition we define a macro FE space

$$
S_{0}^{l}\left(\Omega, \mathcal{T}_{H}\right)=\left\{v^{H} \in H_{0}^{1}(\Omega) ;\left.v^{H}\right|_{K} \in \mathcal{R}^{l}(K), \forall K \in \mathcal{T}_{H}\right\},
$$

where $\mathcal{R}^{l}(K)$ is the space $\mathcal{P}^{l}(K)$ of polynomials on $K$ of total degree at most $l$ if $K$ is a simplicial FE, or the space $\mathcal{Q}^{l}(K)$ of polynomials on $K$ of degree at most $l$ in each variables if $K$ is a quadrilateral FE. Within each macro elements $K \in \mathcal{T}_{H}$ we consider, for $j=1, \ldots, J$,

- integration points $x_{j, K} \in K$,

- sampling domains $K_{\delta_{j}}=x_{j, K}+\delta I$, where $I=(-1 / 2,1 / 2)^{d}$ and $\delta \geq \varepsilon$,

- quadrature weights $\omega_{j, K}$.

Quadrature formula. For any element $K$ of the triangulation the mapping $F_{K}$ we consider is a $C^{1}$-diffeomorphism such that $K=F_{K}(\hat{K})$, where $\hat{K}$ is the reference element. For a given quadrature formula on $\hat{K}$, the quadrature weights and integration points on $K \in \mathcal{T}_{H}$ are given by $x_{j, K}=F_{K}\left(\hat{x}_{j}\right), \omega_{j}=\hat{\omega}_{j, K} \operatorname{det}\left(\partial F_{K}\right), j=1, \ldots, J$. We next state the assumptions that we make on the quadrature formulas. These assumptions (see (Q1), (Q2) below) are the usual assumptions when using the FEM with numerical quadrature in order to preserve the optimal convergence rates ([18, Chap. 4.1]). They read

(Q1) $\hat{\omega}_{j}>0, j=1, \ldots, J, \quad \sum_{j \in J} \hat{\omega}_{j}\left|\nabla \hat{p}\left(\hat{x}_{j}\right)\right|^{2} \geq \hat{\lambda}\|\nabla \hat{p}\|_{L^{2}(\hat{K})}^{2} ;$

(Q2) $\int_{\hat{K}} \hat{q}(x) d x=\sum_{j \in J} \hat{\omega}_{j} \hat{q}\left(\hat{x}_{j}\right), \forall \hat{q}(\hat{x}) \in \mathcal{R}^{\sigma}(\hat{K})$, where $\sigma=\max (2 l-$ $2, l)$ if $\hat{K}$ is a simplicial $\mathrm{FE}$, or $\sigma=\max (2 l-1, l+1)$ if $\hat{K}$ is a rectangular FE.

As an example, for $l=1$ and triangular elements, we can choose $J=1, \omega_{j}=|K|$ and $\hat{x}$ located at the barycenter of $\hat{K}$.

Micro finite element space. In each sampling domain $K_{\delta_{j}}$, the microstructure of the oscillating tensor is tested through a micro FEM. 
We thus consider a (micro) partition $\mathcal{T}_{h}$ of each sampling domain $K_{\delta_{j}}$ made of simplicial or quadrilateral elements $Q$ of diameter $h_{Q}$ and denote $h=\max _{i \in \mathcal{T}_{h}} h_{Q}$. The micro FE space is defined as

$$
S^{q}\left(K_{\delta_{j}}, \mathcal{T}_{h}\right)=\left\{z^{h} \in W\left(K_{\delta_{j}}\right) ;\left.z^{h}\right|_{Q}, \in \mathcal{R}^{r}(Q), Q \in \mathcal{T}_{h}\right\},
$$

where the Sobolev space $W\left(K_{\delta_{j}}\right)$ depends on the boundary conditions used for the micro problems. This determines the coupling condition between macro and micro FE functions. We will consider

$$
W\left(K_{\delta_{j}}\right)=W_{p e r}^{1}\left(K_{\delta_{j}}\right)=\left\{v \in H_{p e r}^{1}\left(K_{\delta_{j}}\right) ; \int_{K_{\delta_{j}}} v d x=0\right\}
$$

for a periodic coupling or

$$
W\left(K_{\delta_{j}}\right)=H_{0}^{1}\left(K_{\delta_{j}}\right)
$$

for a coupling through Dirichlet boundary conditions. Other boundary conditions such as Robin boundary conditions could be used as well. As we resolve the fine scale on $K_{\delta_{j}}$, we have to use a mesh of size $h<\varepsilon$ for the micro partition $\mathcal{T}_{h}$ of $K_{\delta_{j}}$. Remember that the sampling domain $K_{\delta_{j}}$ is of size comparable to $\varepsilon$. Thus the fine scale mesh is only used on a subdomain of the computational domain $\Omega$.

Multiscale Method. Having defined the macro and micro FE spaces, we now introduce a macro bilinear form. Let $v^{H}, w^{H} \in S_{0}^{l}\left(\Omega, \mathcal{T}_{H}\right)$ and consider

$$
B_{H}\left(v^{H}, w^{H}\right):=\sum_{K \in \mathcal{T}_{H}} \sum_{j=1}^{J} \frac{\omega_{j, K}}{\left|K_{\delta_{j}}\right|} \int_{K_{\delta_{j}}} a^{\varepsilon}(x) \nabla v_{K_{j}}^{h} \cdot \nabla w_{K_{j}}^{h} d x,
$$

where $v_{K_{j}}^{h}, w_{K_{j}}^{h}$ represent micro functions defined on the $J$ sampling domains $K_{\delta_{j}}$. These micro functions are defined in the following way. Find $v_{K_{j}}^{h}\left(\right.$ resp. $\left.w_{K_{j}}^{h}\right)$ such that $\left(v_{K_{j}}^{h}-v_{l i n, K_{j}}^{H}\right) \in S_{h}^{q}\left(K_{\delta_{j}}, \mathcal{T}_{h}\right)$ and

$$
\int_{K_{\delta_{j}}} a^{\varepsilon}(x) \nabla v_{K_{j}}^{h} \cdot \nabla z^{h} d x=0 \quad \forall z^{h} \in S_{h}^{q}\left(K_{\delta_{j}}, \mathcal{T}_{h}\right),
$$

where $S_{h}^{q}\left(K_{\delta_{j}}, \mathcal{T}_{h}\right)$ is the micro FE space (3.2) and $v_{l i n, K_{j}}^{H}$ is a linearization around the integration points $x_{j, K}$ which reads

$$
v_{l i n, K_{j}}^{H}=v^{H}\left(x_{j, K}\right)+\left(x-x_{j, K}\right) \cdot \nabla v^{H}\left(x_{j, K}\right) .
$$

The FE-HMM is now given as follows. Find $u^{H} \in S_{0}^{l}\left(\Omega, \mathcal{T}_{H}\right)$ such that

$$
B_{H}\left(u^{H}, v^{H}\right)=\left\langle f, v^{H}\right\rangle \forall v^{H} \in S_{0}^{l}\left(\Omega, \mathcal{T}_{H}\right) .
$$


An efficient and versatile implementation of the FE-HMM has been proposed in [9], and a code is available at http://anmc.epfl.ch.

Semi-discrete FE-HMM. We close this section by introducing the so-called semi-discrete FE-HMM solution. Consider the bilinear form

$$
\bar{B}_{H}\left(v^{H}, w^{H}\right):=\sum_{K \in \mathcal{T}_{H}} \sum_{j=1}^{J} \frac{\omega_{j, K}}{\left|K_{\delta_{j}}\right|} \int_{K_{\delta_{j}}} a^{\varepsilon}(x) \nabla v_{K_{j}} \cdot \nabla w_{K_{j}} d x,
$$

where $v_{K_{j}}, w_{K_{j}}$ are micro solutions constrained by the macro FE functions $v^{H}, w^{H}$, respectively, similarly as above, but obtained from the problem (3.6) in the Sobolev spaces (3.3) or (3.4). The semi-discrete FE-HMM solution is then given by the following problem. Find $\bar{u}^{H} \in S_{0}^{l}\left(\Omega, \mathcal{T}_{H}\right)$ such that

$$
\bar{B}_{H}\left(\bar{u}^{H}, v^{H}\right)=\left\langle f, v^{H}\right\rangle \forall v^{H} \in S_{0}^{l}\left(\Omega, \mathcal{T}_{H}\right) .
$$

It is the error $\left\|u_{0}-\bar{u}^{H}\right\|$ ( $L^{2}$ or $H^{1}$ norm) that has been analyzed in [24]. Of course this solution is usually not available as the exact solution of the micro problem is usually not known.

\section{Preliminaries}

In a priori error analysis bound of the type

$$
\left\|u_{0}-u^{H}\right\| \leq r\left(u_{0}, H, h, \text { data }\right)
$$

are thought, where data represents source and boundary terms. The above error (usually in the $L^{2}$ or $H^{1}$ norm) is estimated by constants involving the (unknown) exact solution. Such bounds are crucial to establish convergence, stability and asymptotic behavior of the error. Furthermore in a multiscale context as for the FE-HMM, a priori estimates give crucial insights on how macro and micro meshes have (a priori) to be refined. Indeed, in view of Theorem 17 of Section 5 we see that

$$
\hat{h} \propto H^{\frac{l}{2 q}}\left(H^{1} \text { norm }\right), \hat{h} \propto H^{\frac{l+1}{2 q}}\left(L^{2} \text { norm }\right),
$$

is an appropriate refinement strategy. In the above equations, $H$ is the size of the macro element and $\hat{h}=\frac{h}{\varepsilon}$, where $h$ is the mesh discretizing the actual sampling domain. Notice that $h=\frac{\delta}{r}$, where $r$ is the number of points per spatial direction; as $\delta=c s t \cdot \varepsilon, \hat{h}=\frac{h}{\varepsilon}=\frac{c s t}{r}$ is independent of $\varepsilon$. The above estimates give a criteria to refine the micro-mesh for a given size of the macro-mesh. For the particular case $l=q=1$ (piecewise linear FEM in the micro and macro FE spaces) denoting $N_{m a c}, N_{m i c}$ the 
total number of macro and micro degrees of freedom, respectively the above criterion reads

$$
N_{m i c} \propto \sqrt{N_{m a c}}\left(H^{1} \text { norm }\right), N_{m i c} \propto N_{m a c} \quad\left(L^{2} \text { norm }\right) .
$$

The important question is now how to quantify the quality or accuracy of an actual solution in order to construct a sequence of meshes that equidistributes the (macroscopic) error and minimize the computational effort.

For problems with e.g. re-entrant corners, cracks or high contrasts in the macroscopic coefficients, a uniform refinement of the macroscopic mesh discretizing the computational domain is known to be quite inefficient and adaptive local refinements based on error indicators are required [13],[34]. While the theory for adaptive refinement is well developed for single-scale problems, the corresponding theory for multiscale methods is still at its infancy. General methodologies for refinements in quantities of interest have been proposed in [31] (see also the references therein). Here we discuss the a posteriori error analysis for the FE-HMM. Such an analysis is based on bounds of the type

$$
\left\|u_{0}-u^{H}\right\| \leq r\left(u^{H}, H, h, \text { data }\right),
$$

depending on the actual computed solution $u^{H}$ and quantifying the quality and local accuracy of the numerical solution. Deriving a strategy able to localize and compute the residual $\left.\eta_{H}(K) \simeq r\left(u^{H}, H, h\right.$, data $)\right|_{K}$ on each element $K \in \mathcal{T}_{H}$ allows to implement a refinement strategy based on the following steps

$$
\text { SOLVE } \rightarrow \text { ESTIMATE } \rightarrow \text { MARK } \rightarrow \text { REFINE. }
$$

The differential equation is solved in the SOLVE procedure and the local accuracy of the obtained numerical solution $u^{H}$ is estimated in the ESTIMATE procedure by evaluating $\eta_{H}(K)$. Based on these indicators, some elements (where the error indicator are the largest) are selected in the MARK procedure and refined in the REFINE procedure. The whole procedure aims at equi-distributing the global error among the elements. As the overall error also depends on the micro error in the sampling domains, appropriate local refinement of the mesh in the sampling domains should be performed in the macro elements marked for refinement. In view of Theorem 21 of Section 6 we see that

$$
\hat{h} \propto \sqrt{H_{K}},
$$

is an appropriate refinement strategy (for the energy norm). Here piecewise linear macro and micro FE are used and $\hat{h}=\frac{h}{\varepsilon}$ is as above. 
A priori and a posteriori analysis for numerical homogenization

Before discussing the a priori and a posteriori error analysis for the FE-HMM, we collect in this section a number of results as a preparation for the a priori and a posteriori error analysis.

\subsection{Standard FEM with numerical quadrature}

We recall the standard FEM for the homogenized problem (2.3) based on the quadrature formulas introduced in Section 3 (see [18, Chap. 4.1]). We consider

$$
B_{0, H}\left(v^{H}, w^{H}\right)=\sum_{K \in \mathcal{T}_{H}} \sum_{j=1}^{J} \omega_{j, K} a^{0}\left(x_{j, K}\right) \nabla v^{H}\left(x_{j, K}\right) \nabla w^{H}\left(x_{j, K}\right),
$$

and define the following problem. Find $u_{0}^{H} \in S_{0}^{l}\left(\Omega, \mathcal{T}_{H}\right)$ such that

$$
B_{0, H}\left(u^{H}, v^{H}\right)=\left\langle f, v^{H}\right\rangle, \forall v^{H} \in S_{0}^{l}\left(\Omega, \mathcal{T}_{H}\right) .
$$

In order to use a FEM with numerical quadrature, some regularity on the tensor $a^{0}(x)$ is required. We assume that for $i, j=1, \ldots, d$,

$$
a_{i j}^{0}(x) \text { is Lipschitz continuous in } K \text { for any } K \in \mathcal{T}_{H} .
$$

\subsection{Energy equivalence and coercivity}

We refer to [1],[7, Sect. 3.3.1] for a discussion and proof of the following two lemmata.

Lemma 1. Let $v^{H} \in S_{0}^{l}\left(\Omega, \mathcal{T}_{H}\right)$ and let $v_{K_{j}}^{h}$ be the corresponding solution of (3.6) in $S^{q}\left(K_{\delta_{j}}, \mathcal{T}_{h}\right)$ with boundary conditions given by (3.3) or (3.4). Then we have

$$
\left\|\nabla v_{l i n}^{H}\right\|_{L^{2}\left(K_{\delta}\right)} \leq\left\|\nabla v_{K_{j}}^{h}\right\|_{L^{2}\left(K_{\delta}\right)} \leq \sqrt{\frac{\Lambda}{\lambda}}\left\|\nabla v_{l i n}^{H}\right\|_{L^{2}\left(K_{\delta}\right)},
$$

where $v_{\text {lin }}^{H}$ is defined in (3.7) and $\lambda, \Lambda$ are defined in (2.2).

From the above we can deduce the coercivity of the bilinear form (3.5) hence the existence and uniqueness of the problem (3.8).

Proposition 2. Assume that (2.2) and (Q1) hold. Then, the bilinear form $B_{H}(\cdot, \cdot)$ defined in (3.5) is elliptic, bounded (independently of $\varepsilon$ ), and

$\gamma\left\|v^{H}\right\|_{H^{1}(\Omega)}^{2} \leq B_{H}\left(v^{H}, v^{H}\right), \quad\left|B_{H}\left(v^{H}, w^{H}\right)\right| \leq \Gamma\left\|v^{H}\right\|_{H^{1}(\Omega)}\left\|w^{H}\right\|_{H^{1}(\Omega)}$,

for all $v^{H}, w^{H} \in S_{0}^{l}\left(\Omega, \mathcal{T}_{H}\right)$, where $\gamma, \Gamma$ are independent of $H, h$ and $\varepsilon$. 
Remark 3. Similarly one shows that the bilinear form $(3.10) \bar{B}_{H}(\cdot, \cdot)$ defined in (3.10) is elliptic and bounded and that the associated microsolutions $v_{K_{j}}$ (obtained from (3.6) in the Sobolev space $W\left(K_{\delta_{j}}\right)$ ) satisfy the inequality (4.3).

\subsection{Micro problem, coupling condition and micro er- ror}

The following construction of a numerically homogenized tensor will be useful for the analysis (see [8],[10] for details).

Let $\mathbf{e}_{\mathbf{i}}, i=1, \ldots, d$ denote the canonical basis $\mathbb{R}^{d}$. For each $\mathbf{e}_{\mathbf{i}}$ we consider the following problem: find $\psi_{K_{j}}^{i, h} \in S^{q}\left(K_{\delta_{j}}, \mathcal{I}_{h}\right)$ such that

$$
\int_{K_{\delta_{j}}} a^{\varepsilon}(x) \nabla \psi_{K_{j}}^{i, h} \nabla z^{h} d x=-\int_{K_{\delta_{j}}} a^{\varepsilon}(x) \mathbf{e}_{\mathbf{i}} \cdot \nabla z^{h} d x, \forall z^{h} \in S^{q}\left(K_{\delta_{j}}, \mathcal{T}_{h}\right),
$$

where $S^{q}\left(K_{\delta_{j}}, \mathcal{T}_{h}\right)$ is defined in (3.2) with either periodic or Dirichlet boundary conditions. We also consider the problem: find $\psi_{K_{j}}^{i} \in W\left(K_{\delta_{j}}\right)$ such that

$$
\int_{K_{\delta_{j}}} a^{\varepsilon}(x) \nabla \psi_{K_{j}}^{i} \nabla z d x=-\int_{K_{\delta_{j}}} a^{\varepsilon}(x) \mathbf{e}_{\mathbf{i}} \cdot \nabla z d x, \forall z \in W\left(K_{\delta_{j}}\right),
$$

where the Sobolev space $W\left(K_{\delta_{j}}\right)$ is defined in (3.3) or (3.4). For the analysis of the micro problems, regularity results are needed. Motivated by the case of periodic tensors (e.g. tensors of the form $a^{\varepsilon}=a(x, x / \varepsilon)$ ) we state the following regularity assumption on the solution of the problem (4.6):

(H2) for $q \in \mathbb{N}$ we assume that $\left|\psi_{K_{j}}^{i}\right|_{H^{q+1}\left(K_{\delta_{j}}\right)} \leq C \varepsilon^{-q} \sqrt{\left|K_{\delta_{j}}\right|}$, with $C$ independent of $\varepsilon$, of the quadrature points $x_{j, K}$ and the domain $K_{\delta_{j}}$.

Remark 4. Without further knowledge of the structure of the oscillating tensor $a^{\varepsilon}$, we will impose Dirichlet boundary conditions for (4.5). If one assumes that $a^{\varepsilon}$ is smooth, e.g., $a^{\varepsilon} \in W^{1, \infty}(\Omega)$ and that $\left|a_{i j}^{\varepsilon}\right|_{L^{\infty}(\Omega)} \leq$ $C \varepsilon^{-1}$ for $i, j=1, \ldots, d$, then $(\mathbf{H 2})$ can be proved for $q=1$ following classical $H^{2}$ regularity results ([27, Chap. 2.6]) (for $q=0$ (H2) can be established by following the lines of the proof of Lemma 1). If $a^{\varepsilon}=$ $a(x, x / \varepsilon)=a(x, y) \quad Y$-periodic in $y$ and $\delta / \varepsilon \in \mathbb{N}$, then (H2) can be established for a given $q$, provided that the tensor $a^{\varepsilon}$ is smooth enough, following classical regularity results for solutions of periodic boundary value problems (see [16, Chap. 3] and also [5]).

We then define two tensors

$$
a_{K}^{0}\left(x_{j, K}\right)=\frac{1}{\left|K_{\delta_{j}}\right|} \int_{K_{\delta_{j}}} a^{\varepsilon}(x)\left(I+J_{\psi_{K_{j}}^{h}(x)}^{T}\right) d x
$$


A priori and a posteriori analysis for numerical homogenization 11 where $J_{\psi_{K_{j}}^{h}(x)}$ is a $d \times d$ matrix with entries $\left(J_{\psi_{K_{j}}^{h}(x)}\right)_{i \ell}=\left(\partial \psi_{K_{j}}^{i, h}\right) /\left(\partial x_{\ell}\right)$ and

$$
\bar{a}_{K}^{0}\left(x_{j, K}\right)=\frac{1}{\left|K_{j}\right|} \int_{K_{j}} a^{\varepsilon}(x)\left(I+J_{\psi_{K_{j}}(x)}^{T}\right) d x,
$$

where $J_{\psi_{K_{j}}(x)}$ is a $d \times d$ matrix with entries $\left(J_{\psi_{K_{j}}(x)}\right)_{i \ell}=\left(\partial \psi_{K_{j}}^{i}\right) /\left(\partial x_{\ell}\right)$.

If we assume more specific spatial structure of the oscillating tensor as for example

(H3)

$$
\begin{aligned}
& a^{\varepsilon}(x)=a(x, x / \varepsilon)=a(x, y) \quad Y \text {-periodic in } y, \\
& a_{i j}(x, y) \in \mathcal{C}\left(\bar{\Omega} ; W_{p e r}^{1, \infty}(Y)\right), \text { for all } i, j=1, \ldots, d,
\end{aligned}
$$

where we set $Y=(0,1)^{d}$ for simplicity, then we can estimate the so-called modeling error (see Lemmata 7 and 8)

$$
\sup _{K \in \mathcal{T}_{H}, x_{j, K} \in K}\left\|a^{0}\left(x_{j, K}\right)-\bar{a}_{K}^{0}\left(x_{j, K}\right)\right\|_{F},
$$

where $a^{0}\left(x_{j, K}\right)$ is the homogenized tensor evaluated at the quadrature points of $K \in \mathcal{T}_{H}$.

Remark 5. In what follows we will always assume that $\psi_{K_{j}}^{i}$ and $\psi_{K_{j}}^{i, h}$ (the solutions of (4.5) and (4.6), respectively) are solved with the same boundary conditions, i.e., either $\psi_{K_{j}}^{i} \in H_{0}^{1}\left(K_{\delta_{j}}\right)$ and $\psi_{K_{j}}^{i, h} \in S^{q}\left(K_{\delta_{j}}, \mathcal{T}_{h}\right) \subset$ $H_{0}^{1}\left(K_{\delta_{j}}\right)$ or $\psi_{K_{j}}^{i} \in W_{p e r}^{1}\left(K_{\delta_{j}}\right)$ and $\psi_{K_{j}}^{i, h} \in S^{q}\left(K_{\delta_{j}}, \mathcal{T}_{h}\right) \subset W_{p e r}^{1}\left(K_{\delta_{j}}\right)$.

The next Lemma can be proved following the lines of [8, Lemma 5.1] (see also [7, Lemma 10]).

Lemma 6. Let $\psi_{K_{j}}^{i}, \psi_{K_{j}}^{i, h}$ be the solutions of (4.5) and (4.6), respectively. Assume that $W\left(K_{\delta_{j}}\right)=H_{0}^{1}\left(K_{\delta_{j}}\right)$ and that (H2) holds. Alternatively, assume that $(\mathbf{H 2})$ and $(\mathbf{H 3})$ hold, that $W\left(K_{\delta_{j}}\right)=W_{\text {per }}^{1}\left(K_{\delta_{j}}\right)$ and that $\delta / \varepsilon \in \mathbb{N}$. Then

$$
\sup _{K \in \mathcal{T}_{H}, x_{j, K} \in K}\left\|\bar{a}^{0}\left(x_{j, K}\right)-a_{K}^{0}\left(x_{j, K}\right)\right\|_{F} \leq C\left(\frac{h}{\varepsilon}\right)^{2 q},
$$

where $C$ is independent of $h$ and $\varepsilon$.

The following two lemmata have been proved in [7],[8].

Lemma 7. Let $\psi_{K_{j}}^{i}, \psi_{K_{j}}^{i, h}$ be the solutions of (4.5) and (4.6), respectively. Assume that $W\left(K_{\delta_{j}}\right)=W_{\text {per }}^{1}\left(K_{\delta_{j}}\right)$, that $\delta / \varepsilon \in \mathbb{N}$ and (H3) hold. Then

$$
\sup _{K \in \mathcal{T}_{H}, x_{j, K} \in K}\left\|a^{0}\left(x_{j, K}\right)-\bar{a}_{K}^{0}\left(x_{j, K}\right)\right\|_{F} \leq C \delta,
$$

where $C$ is independent of $\varepsilon$. 
Notice that the constant in the Lemma 7 depends on the quantities $\max _{x \in \bar{\Omega}}\left\|a_{i, j}(x, \cdot)\right\|_{W_{\text {per }}^{1, \infty}(Y)} \quad i, j=1, \ldots, d$.

If the decomposition $a(x, x / \varepsilon)$ is explicitly known one can define a slightly modified FE-HMM by collocating the slow variable of the tensor $a\left(x_{j, K}, x / \varepsilon\right)$ at the quadrature points $x_{j, K}$ in the problem (3.6) and in the bilinear form (3.5). Correspondingly, one collocates the slow variables at the quadrature points $x_{j, K}$ in the semi-discrete problem (4.6). We then obtain

Lemma 8. If along with the hypothesis of Lemma 7, we assume a tensor collocated at the integration points $a\left(x_{j, K}, x / \varepsilon\right)$ in the problem (4.6). Then

$$
\sup _{K \in \mathcal{T}_{H}, x_{j, K} \in K}\left\|a^{0}\left(x_{j, K}\right)-\bar{a}_{K}^{0}\left(x_{j, K}\right)\right\|_{F} \equiv 0 .
$$

If we do not assume that the sampling domain size is an integer number of the " $\varepsilon$ period" we have the following result proved in [24].

Lemma 9. Suppose $\psi_{K_{j}}^{i}$ is the solution of the cell problem (4.6) in the space $W\left(K_{\delta_{j}}\right)=H_{0}^{1}\left(K_{\delta_{j}}\right)$, that $\delta / \varepsilon \notin \mathbb{N}$ and (H3) hold. Then

$$
\sup _{K \in \mathcal{T}_{H}, x_{j, K} \in K}\left\|a^{0}\left(x_{j, K}\right)-\bar{a}_{K}^{0}\left(x_{j, K}\right)\right\|_{F} \leq C\left(\delta+\frac{\varepsilon}{\delta}\right) .
$$

\subsection{Multiscale flux for non-conforming FEs and a posteriori estimates}

When attempting to derive a posteriori error estimates or when dealing with nonconforming macroscopic spaces, elementwise integration by parts is needed. This leads to fluxes on the edges of the elements. Such quantities are not readily available from the FE-HMM. This issue can be overcome by the introduction of so-called multiscale fluxes, first proposed in [6]. We describe the procedure for a quadrature formula with $J=1$ (see Section 3) and simplicial elements, but emphasize that generalizations to more general quadrature formulas could be derived. For simplicity we skip here the index related to the quadrature point and simply write $K_{\delta}^{ \pm}$ instead of $K_{\delta_{1}}^{ \pm}, v_{K}^{h}$ instead of $v_{K_{1}}^{h}$ or $a_{K}^{0}\left(x_{K}\right)$ instead of $a_{K}^{0}\left(x_{K_{1}}\right)$.

Let $K^{+}, K^{-} \in \mathcal{T}_{H}$ be elements sharing a common interface $e \in$ $\mathcal{E}_{H}$, where $\mathcal{E}_{H}$ denotes the set of interfaces. For both elements we consider the associated sampling domains $K_{\delta}^{+}$and $K_{\delta}^{-}$, and the associated microfunctions $v_{K^{+}}^{h}$ and $v_{K^{-}}^{h}$, solutions of the microproblems (3.6). We then introduce the following multiscale flux

$$
\begin{aligned}
& \llbracket \overline{a^{\varepsilon}(x) \nabla v_{K}^{h}} \rrbracket_{e} \\
& :=\left(\frac{1}{\left|K_{\delta}^{+}\right|} \int_{K_{\delta}^{+}} a^{\varepsilon}(x) \nabla v_{K^{+}}^{h} d x-\frac{1}{\left|K_{\delta}^{-}\right|} \int_{K_{\delta}^{-}} a^{\varepsilon}(x) \nabla v_{K^{-}}^{h} d x\right) \cdot n_{e}
\end{aligned}
$$


A priori and a posteriori analysis for numerical homogenization

for $e \notin \partial \Omega$, and $\llbracket \overline{a^{\varepsilon}(x) \nabla v_{K}^{h}} \rrbracket_{e}:=0$ if $e \in \partial \Omega$. Here we choose the unit outward normal $n_{e}$ to be $n^{+}$. Similarly as in the Lemma 12 below, one can prove that

$$
\frac{1}{\left|K_{\delta}\right|} \int_{K_{\delta}} a^{\varepsilon}(x) \nabla v_{K}^{h} d x=a_{K}^{0}\left(x_{K}\right) \nabla v_{K}^{H}
$$

Then, following [10, Lemma 9] one can show

Lemma 10. Consider $a_{K}^{0}(\cdot)$ defined in (4.7) and $v^{H} \in S_{0}^{1}\left(\Omega, \mathcal{T}_{H}\right)$. Let $v_{K}^{h}$ be such that $\left(v_{K}^{h}-v^{H}\right) \in S_{h}^{1}\left(K_{\delta}, \mathcal{T}_{h}\right)$ and obtained from the problem (3.6). Then we have the following identity

$$
\sum_{K \in \mathcal{T}_{H}} \int_{K} a_{K}^{0}\left(x_{K}\right) \nabla v^{H} \nabla w d x=\sum_{e \in \mathcal{E}} \int_{e} \llbracket \overline{a^{\varepsilon}(x) \nabla v_{K}^{h}} \rrbracket_{e} w d s,
$$

for all $w \in H_{0}^{1}(\Omega)$.

Lemma 11. For all $v \in H_{0}^{1}(\Omega)$, we have

$$
\begin{aligned}
& B_{0}\left(u_{0}-u^{H}, v\right)=\int_{\Omega} f v d x-\sum_{e \in \mathcal{E}} \int_{e} \llbracket \overline{a^{\varepsilon}(x) \nabla u_{K}^{h}} \rrbracket_{e} v d s \\
& +\sum_{K \in \mathcal{T}_{H}} \int_{K}\left(a_{K}^{0}\left(x_{K}\right)-a^{0}(x)\right) \nabla u^{H} \nabla v d x,
\end{aligned}
$$

where $u_{0}$ is the solution of (2.3), $u^{H}$ is the solution of (3.8) and $u_{K}^{h}$ the corresponding micro functions, solutions of (3.6) on each sampling domain. The multiscale flux $\llbracket \overline{a^{\varepsilon}(x) \nabla u_{K}^{h}} \rrbracket_{e}$ is defined in (4.13).

The following well-known results will be used for a posteriori analysis and are recalled for convenience.

Interpolation, trace and inverse estimates. The so-called Clément interpolation operator (see [20]) is a linear operator $I_{H}: H^{1}(\Omega) \rightarrow$ $S_{0}^{1}\left(\Omega, \mathcal{T}_{H}\right)$ having the property that for all $v \in H^{1}(\Omega)$ and $K \in \mathcal{T}_{H}$

$$
\left\|v-I_{H} v\right\|_{L^{2}(K)} \leq C H_{K}\|\nabla v\|_{L^{2}(N(K))}
$$

and

$$
\left\|\nabla\left(v-I_{H} v\right)\right\|_{L^{2}(K)} \leq C\|\nabla v\|_{L^{2}(N(K))},
$$

where $N(K)$ is a neighborhood of $K$ that consists of all elements of $\mathcal{T}_{H}$ which have a non-empty intersection with $K$.

The following trace inequality (see for example [12, Thm. 3.10]) is useful. For an element $K_{e}$ of the triangulation $\mathcal{T}_{H}$ with sides $e \in \mathcal{E}_{H}$ and $v \in H^{1}\left(K_{e}\right)$ we have

$$
\|v\|_{L^{2}(e)} \leq C H_{e}^{1 / 2}\|\nabla v\|_{L^{2}\left(K_{e}\right)}+C H_{e}^{-1 / 2}\|v\|_{L^{2}\left(K_{e}\right)} .
$$


Combining the Clément interpolation estimates and the trace inequality gives

$$
\begin{aligned}
\left\|v-I_{H} v\right\|_{L^{p}(e)} & \leq C H_{K}^{1 / 2}\left\|\nabla\left(v-I_{H} v\right)\right\|_{L^{2}(K)}+C H_{K}^{-1 / 2}\left\|v-I_{H} v\right\|_{L^{2}(K)} \\
& \leq C H_{K}^{1 / 2}\|\nabla v\|_{L^{2}(N(K))} .
\end{aligned}
$$

Finally, we recall the following inverse inequality (see for example [18])

$$
\left\|\nabla v^{H}\right\|_{L^{2}(K)} \leq C H_{K}^{-1}\left\|v^{H}\right\|_{L^{2}(K)},
$$

for $v^{H} \in S_{0}^{1}\left(\Omega, \mathcal{T}_{H}\right)$.

\subsection{Reformulation of the FE-HMM}

The following two lemmata allow us to reformulate the FE-HMM. We emphasize here that this reformulation will only be used for analysis. The original formulation is more convenient to implement as one can make use of the standard structure of a FE code (see [9]). The following lemma has been proved in [8].

Lemma 12. Consider $a_{K}^{0}(\cdot)$ defined in (4.7) and $v^{H}, w^{H} \in S_{0}^{l}\left(\Omega, \mathcal{T}_{H}\right)$. Let $v_{K_{j}}^{h}\left(\right.$ resp. $\left.w_{K_{j}}^{h}\right)$ be such that $\left(v_{K_{j}}^{h}-v_{\text {lin }}^{H}\right) \in S_{h}^{q}\left(K_{\delta_{j}}, \mathcal{T}_{h}\right)$ and obtained from the problem (3.6), where $v_{\text {lin }}^{H}\left(\right.$ resp. $\left.w_{\text {lin }}^{H}\right)$ are defined in (3.7). Then, we have the following identity

$$
\frac{1}{\left|K_{\delta_{j}}\right|} \int_{K_{\delta_{j}}} a^{\varepsilon}(x) \nabla v_{K_{j}}^{h} \cdot \nabla w_{K_{j}}^{h} d x=\frac{1}{|K|} \int_{K} a_{K}^{0}\left(x_{j, K}\right) \nabla v_{l i n}^{H} \cdot \nabla w_{l i n}^{H} d x .
$$

Similarly we have

Lemma 13. Consider $\bar{a}_{K}^{0}(\cdot)$ defined in (4.8) and $v^{H}, w^{H} \in S_{0}^{l}\left(\Omega, \mathcal{T}_{H}\right)$. Let $v_{K_{j}}$ (resp. $w_{K_{j}}$ ) be such that $\left(v_{K_{j}}-v_{\text {lin }}^{H}\right)$ is in the Sobolev space $W\left(K_{\delta}\right)$ (defined in (3.3) or (3.4)) and obtained from the problem (3.6) (in $\left.W\left(K_{\delta}\right)\right)$, where $v_{\text {lin }}^{H}\left(\right.$ resp. $\left.w_{\text {lin }}^{H}\right)$ are defined in (3.7). Then, we have the following identity

$$
\frac{1}{\left|K_{\delta_{j}}\right|} \int_{K_{\delta_{j}}} a^{\varepsilon}(x) \nabla v_{K_{j}} \cdot \nabla w_{K_{j}} d x=\frac{1}{|K|} \int_{K} \bar{a}_{K}^{0}\left(x_{j, K}\right) \nabla v_{l i n}^{H} \cdot \nabla w_{l i n}^{H} d x .
$$

In view of the two above lemmata we can reformulate the FE-HMM bilinear form $B_{H}\left(v^{H}, w^{H}\right)$ defined in (3.5) as

$$
B_{H}\left(v^{H}, w^{H}\right)=\sum_{K \in \mathcal{T}_{H}} \sum_{j=1}^{J} \omega_{j, K} a_{K}^{0}\left(x_{j, K}\right) \nabla v^{H}\left(x_{j, K}\right) \cdot \nabla w^{H}\left(x_{j, K}\right),
$$


A priori and a posteriori analysis for numerical homogenization

and the semi-discrete FE-HMM bilinear form $\bar{B}_{H}\left(v^{H}, w^{H}\right)$ defined in $(3.9)$ as

$$
\bar{B}_{H}\left(v^{H}, w^{H}\right)=\sum_{K \in \mathcal{T}_{H}} \sum_{j=1}^{J} \omega_{j, K} \bar{a}_{K}^{0}\left(x_{j, K}\right) \nabla v^{H}\left(x_{j, K}\right) \cdot \nabla w^{H}\left(x_{j, K}\right) .
$$

\section{A priori error analysis}

In this section we discuss the a priori analysis for our multiscale numerical method. To proceed, we start with the following decomposition into so-called macro error $\left(e_{M A C}\right)$, modeling error $\left(e_{M O D}\right)$ and micro error $\left(e_{M I C}\right)$

$$
\left\|u_{0}-u^{H}\right\| \leq \underbrace{\left\|u_{0}-u_{0}^{H}\right\|}_{e_{M A C}}+\underbrace{\left\|u_{0}^{H}-\bar{u}^{H}\right\|}_{e_{M O D}}+\underbrace{\left\|\bar{u}^{H}-u^{H}\right\|}_{e_{M I C}},
$$

where $\|\cdot\|$ denotes the $L^{2}$ or the $H^{1}$ norm, and $u_{0}, u_{0}^{H}, \bar{u}^{H}, u^{H}$ denote the solutions of the problems $(2.3),(4.2),(3.10),(3.8)$, respectively.

Proposition 14 (Macro error). Let $u_{0}, u_{0}^{H}$ be the solutions of problems (2.3),(4.2), respectively. Suppose that (Q1), (Q2) and (H1) hold and that the homogenized problem has the regularity $u_{0} \in H^{l+1}(\Omega)$. Then we have the following estimates

$$
\begin{aligned}
& \left\|u_{0}-u_{0}^{H}\right\|_{H^{1}(\Omega)} \leq C H^{l}, \\
& \left\|u_{0}-u_{0}^{H}\right\|_{L^{2}(\Omega)} \leq C H^{l+1},
\end{aligned}
$$

where $C$ is independent of $H$ and $h$.

Proof. Follows the classical results for FEMs with numerical quadrature (see [17],[18, Chap. 4.1]).

Proposition 15 (Modelling error). Let $u_{0}^{H}, \bar{u}^{H}$ be the solutions of problems (4.2) and (3.10), respectively. Then we have

$$
\left\|u_{0}^{H}-\bar{u}^{H}\right\|_{H^{1}(\Omega)} \leq C \sup _{K \in \mathcal{T}_{H}, x_{j, K} \in K}\left\|a^{0}\left(x_{j, K}\right)-\bar{a}_{K}^{0}\left(x_{j, K}\right)\right\|_{F},
$$

where $C$ is independent of $H$ and $\varepsilon$.

Proof. Denoting by $w^{H}=u_{0}^{H}-\bar{u}^{H}$, and using the coercivity of the bilinear form $\bar{B}_{H}(\cdot, \cdot)$ (see Remark (3)) we obtain

$C\left\|u_{0}^{H}-\bar{u}^{H}\right\|_{H^{1}(\Omega)}^{2} \leq \bar{B}_{H}\left(u_{0}^{H}-\bar{u}^{H}, w^{H}\right)=\bar{B}_{H}\left(u_{0}^{H}, w^{H}\right)-B_{0, H}\left(u_{0}^{H}, w^{H}\right)$, 
thus

$$
\left\|u_{0}^{H}-\bar{u}^{H}\right\|_{H^{1}(\Omega)} \leq C \sup _{w^{H} \in S_{0}^{l}\left(\Omega, \mathcal{T}_{H}\right)} \frac{\left|\bar{B}_{H}\left(u_{0}^{H}, w^{H}\right)-B_{0, H}\left(u_{0}^{H}, w^{H}\right)\right|}{\left\|w^{H}\right\|_{H^{1}(\Omega)}} .
$$

In view of (4.1) and (4.23) using Cauchy-Schwarz inequality gives (5.4).

Proposition 16 (Micro error). Let $u^{H}, \bar{u}^{H}$ be the solutions of problems (3.8) and (3.10), respectively. Then we have

$$
\left\|\bar{u}^{H}-u^{H}\right\|_{H^{1}(\Omega)} \leq C \sup _{K \in \mathcal{T}_{H}, x_{j, K} \in K}\left\|\bar{a}_{K}^{0}\left(x_{j, K}\right)-a_{K}^{0}\left(x_{j, K}\right)\right\|_{F},
$$

where $C$ is independent of $H, h$ and $\varepsilon$.

Proof. Following the proof of Proposition 15, with $u^{H}$ in the role of $\bar{u}^{H}$ and $\bar{u}^{H}$ in the role of $u_{0}^{H}$, using $B_{H}(\cdot, \cdot)$ instead of $\bar{B}_{H}(\cdot, \cdot)$ and $\bar{B}_{H}(\cdot, \cdot)$ instead of $B_{0, H}(\cdot, \cdot)$ we obtain (5.5).

We can now state the main theorem of this section. To simplify the notation we set

$$
r_{M O D}:=\sup _{K \in \mathcal{T}_{H}, x_{j, K} \in K}\left\|a^{0}\left(x_{j, K}\right)-\bar{a}_{K}^{0}\left(x_{j, K}\right)\right\|_{F},
$$

and

$$
r_{M I C}:=\sup _{K \in \mathcal{T}_{H}, x_{j, K} \in K}\left\|\bar{a}_{K}^{0}\left(x_{j, K}\right)-a_{K}^{0}\left(x_{j, K}\right)\right\|_{F} .
$$

In the theorem below, all the constants are independent of $H, h$ and $\varepsilon$.

Theorem 17. Let $u_{0}, u^{H}$ be the solutions of problems (2.3) and (3.8), respectively. Suppose that $u_{0} \in H^{l+1}(\Omega)$ and that (Q1), (Q2) and (H1) hold (see Sections 3 and 4.1). Then we have

$$
\begin{aligned}
& \left\|u_{0}-u^{H}\right\|_{H^{1}(\Omega)} \leq C_{1} H^{l}+C\left(r_{M I C}+r_{M O D}\right), \\
& \left\|u_{0}-u^{H}\right\|_{L^{2}(\Omega)} \leq C_{1} H^{l+1}+C\left(r_{M I C}+r_{M O D}\right) .
\end{aligned}
$$

If in addition to (Q1), (Q2) and (H1), (H2) hold and $S^{q}\left(K_{\delta_{j}}, \mathcal{T}_{h}\right) \subset$ $H_{0}^{1}\left(K_{\delta_{j}}\right)$, then

$$
\begin{aligned}
& \left\|u_{0}-u^{H}\right\|_{H^{1}(\Omega)} \leq C_{1} H^{l}+C_{2}\left(\frac{h}{\varepsilon}\right)^{2 q}+C r_{M O D}, \\
& \left\|u_{0}-u^{H}\right\|_{L^{2}(\Omega)} \leq C_{1} H^{l+1}+C_{2}\left(\frac{h}{\varepsilon}\right)^{2 q}+C r_{M O D} .
\end{aligned}
$$

Alternatively, we also have (5.10) and (5.11) if (Q1), (Q2), (H1), (H2) and (H3) hold, $\delta / \varepsilon \in \mathbb{N}$ and $S^{q}\left(K_{\delta_{j}}, \mathcal{T}_{h}\right) \subset W_{\text {per }}^{1}\left(K_{\delta_{j}}\right)$. 
If in addition to (Q1),(Q2),(H1) and (H2), (H3) holds, then (5.10) and (5.11) hold with $r_{M O D}$ given by

$$
r_{M O D}:= \begin{cases}C_{3} \delta & \text { if } \delta / \varepsilon \in \mathbb{N}, S^{q}\left(K_{\delta_{j}}, \mathcal{T}_{h}\right) \subset W_{p e r}^{1}\left(K_{\delta_{j}}\right) ; \\ 0 & \text { if } \delta / \varepsilon \in \mathbb{N} \text { and } S^{q}\left(K_{\delta_{j}}, \mathcal{T}_{h}\right) \subset W_{p e r}^{1}\left(K_{\delta_{j}}\right), \text { the } \\ & \text { explicit decomposition } a^{\varepsilon}(x)=a(x, x / \varepsilon) \text { is } \\ & \text { available and collocation in the slow variable } \\ & \text { is used for the } F E-H M M ; \\ C_{4}\left(\delta+\frac{\varepsilon}{\delta}\right) & \text { if } \delta / \varepsilon \notin \mathbb{N} \text { and } S^{q}\left(K_{\delta_{j}}, \mathcal{T}_{h}\right) \subset H_{0}^{1}\left(K_{\delta_{j}}\right) .\end{cases}
$$

Proof. The estimates (5.8) and (5.9) are obtained from the decomposition (5.1) and the Propositions 14,15,16. The estimates (5.10),(5.11) are obtained from the decomposition (5.1), the Propositions 14,15,16 and Lemma 6. Finally the estimates (5.12) are obtained from the decomposition (5.1), the Propositions 14,15,16, Lemma 6 and Lemmata $7,8,9$.

We emphasize that the value of $q$ in the micro error depends on the regularity of the solutions of the micro problem and the chosen boundary conditions (see Remark 4). We close this section by noting that approximation of the fine scale solution $u_{\varepsilon}$ is possible with the FE-HMM by extending the micro information available on the sampling domains $K_{\delta_{j}}$ on the whole corresponding macro element. We refer to [1],[7],[24] for details.

\section{A posteriori error analysis}

Various approaches have been developed for the a posteriori error analysis of single scale problems. We mention estimators based on local residuals, on local boundary value problems or on local superconvergence properties (see [34],[13] for an overview and references). Estimates in terms of quantities of interests or goal oriented adaptivity has also attracted growing attention in the past few years. Such error estimations allow to adapt the finite element mesh to a quantity of interest dictated by the specific application [13],[31]. In this paper we restrict ourself to estimates in the energy norm. We note however that estimates in the energy norm are usually an essential ingredient for estimation in other quantities of interests.

Among the various approaches to a posteriori error analysis (see the discussion in Section 4), we will focus on explicit residual based estimators.

Single-scale adaptivity. Assuming first that the homogenized problem 
(2.3) is explicitly known, then the classical procedure can be described as follows. Consider for $v \in H_{0}^{1}(\Omega)$

$B_{0}\left(u_{0}, v\right)-B_{0}\left(u_{0}^{H}, v\right)=\sum_{K \in \mathcal{T}_{H}} \int_{K}\left(f+\operatorname{div}\left(a^{0}(x) \nabla u_{0}^{H}\right)\right) v d x=\left\langle R\left(u_{0}^{H}\right), v\right\rangle$,

were the residual $R\left(u_{0}^{H}\right)$ is defined as an element of the dual space of $H_{0}^{1}(\Omega)$. A simple calculation shows that

$$
\frac{\lambda}{1+C_{\Omega}^{2}}\left\|u_{0}-u_{0}^{H}\right\|_{H^{1}(\Omega)} \leq\left\|R\left(u_{0}^{H}\right)\right\|_{H^{-1}(\Omega)} \leq \Lambda\left\|u_{0}-u_{0}^{H}\right\|_{H^{1}(\Omega)},
$$

where $\lambda, \Lambda$ are given by (2.2) and $C_{\Omega}$ is the constant given by the Poincaré inequality. The task is thus, for "single scale adaptivity", to find explicit computable (local) estimates for this dual norm [34]. A crucial quantity in these explicit bounds are the so-called fluxes on the interface of elements. Let $K^{+}, K^{-} \in \mathcal{T}_{H}$ be elements sharing a common interface $e \in \mathcal{E}_{H}$, where $\mathcal{E}_{H}$ denotes the set of interfaces. Then

$$
J_{e}\left(u_{0}^{H}\right)=\left(a^{0}(x) \nabla u_{0}^{H,+}-a^{0}(x) \nabla u_{0}^{H,-}\right) \cdot n_{e}=\llbracket a^{0}(x) \nabla u_{0}^{H} \rrbracket_{e},
$$

is the flux at the interface $e$ of an interior edge, where the unit outward normal $n_{e}$ is chosen to be $n^{+}$.

Multiscale adaptivity. As $a^{0}(x)$ is usually not readily available, and as we would like to use only quantities already computed on the sampling domains (to avoid supplementary costs), we have to proceed in a different way.

For simplicity, we use piecewise linear macro an micro FE simplicial elements (i.e. $S_{0}^{1}\left(\Omega, \mathcal{T}_{H}\right)$, and $S^{1}\left(K_{\delta}, \mathcal{T}_{h}\right)$, respectively). Thus, we set $J=1$ in the quadrature formula of the FE-HMM $x_{j, K}=x_{K}$ for the integration node located at the barycenter and $\omega_{j, K}=\omega_{K}=|K|$ (see Section 3). Notice that in this situation, we have $v_{\text {lin }, K}^{H}=v^{H}$ in (3.6) for $v^{H} \in S_{0}^{1}\left(\Omega, \mathcal{T}_{H}\right)$.

Before proceeding with the analysis, we define our local indicators. Consider $f_{H}$, a piecewise constant approximation of $f$ over $\mathcal{T}_{H}$. We then define a local error indicator $\eta_{H}(K)$ on an element $K$ by

$$
\eta_{H}(K)^{2}:=H_{K}^{2}\left\|f_{H}\right\|_{L^{2}(K)}^{2}+\frac{1}{2} \sum_{e \subset \partial K} H_{e}\left\|\overline{\llbracket \overline{a^{\varepsilon} \nabla u_{K}^{h}} \rrbracket_{e}}\right\|_{L^{2}(e)}^{2},
$$

where $\llbracket \overline{a^{\varepsilon} \nabla u^{h}} \rrbracket_{e}$ is defined in (4.13). It is $\eta_{H}(K)$, involving only known quantities (after the numerical solution has been computed), which will be used as an indicator for the a posteriori error. The magnitude of $\eta_{H}(K)$ on each element $K$ will determine whether or not a macro element is 
refined. A possible marking strategy is the so-called Dörfler bulk-chasing procedure [22]. Given a parameter $0<\theta<1$, the procedure consists in finding a minimal subset $\widehat{\mathcal{T}}_{H}$ of $\mathcal{T}_{H}$ such that

$$
\sum_{K \in \widehat{\mathcal{T}}_{H}} \eta_{H}(K)^{2} \geq \theta \eta_{H}(\Omega)^{2} .
$$

The elements in $\widehat{\mathcal{T}}_{H}$ are then marked for refinement.

We also define the data approximation error $\xi_{H}(K)$ on an element $K$ by

$$
\xi_{H}(K)^{2}:=H_{K}^{2}\left\|f_{H}-f\right\|_{L^{2}(K)}^{2}+\left\|\left(a_{K}^{0}\left(x_{K}\right)-a^{0}(x)\right) \nabla u^{H}\right\|_{L^{2}(K)}^{2} .
$$

Here $a^{0}(x)$ is the unknown homogenized tensor of problem (2.3) and $a_{K}^{0}\left(x_{K}\right)$ is the numerical tensor (piecewise constant on each $K$ in the present situation) defined in (4.7). In order to motivate the above error indicator and data approximation error let us give the following lemma.

Lemma 18. Assume that the tensor $a^{\varepsilon}(x)=a(x / \varepsilon)=a(y)$ is $Y$-periodic in $y$ and that the micro problems (3.6) are solved exactly in the FE-HMM, then

$$
\begin{aligned}
& \eta_{H}(K)^{2}=H_{K}^{2}\left\|f_{H}\right\|_{L^{2}(K)}^{2}+\frac{1}{2} \sum_{e \subset \partial K} H_{e}\left\|\llbracket a^{0} \nabla u^{H} \rrbracket_{e}\right\|_{L^{2}(e)}^{2}, \\
& \xi_{H}(K)^{2}=H_{K}^{2}\left\|f_{H}-f\right\|_{L^{2}(K)}^{2} .
\end{aligned}
$$

Proof. The fact that

$$
\llbracket \overline{a^{\varepsilon} \nabla u_{K}} \rrbracket_{e}=\llbracket a^{0} \nabla u^{H} \rrbracket_{e},
$$

with $u_{K}$ being the solution of (3.6) in $W_{p e r}^{1}\left(K_{\delta}\right)$ constrained by $u^{H}$ is a consequence of (4.14) (remember that if $a^{\varepsilon}(x)=a(x / \varepsilon)$ then $a^{0}$ is a constant tensor). The fact that

$$
\left\|\left(a_{K}^{0}\left(x_{K}\right)-a^{0}(x)\right) \nabla u^{H}\right\|_{L^{2}(K)}^{2} \equiv 0
$$

is a consequence of $\sup _{K \in \mathcal{T}_{H}}\left\|\bar{a}^{0}\left(x_{K}\right)-a_{K}^{0}\left(x_{K}\right)\right\|_{F} \equiv 0$ (as (4.9) vanishes with exact micro functions) and (4.11).

The indicator (6.4) and the local data approximation (6.5) are the quantities used in usual (single scale) adaptive FEM based on explicit residual, i.e., the indicator and the data approximation that are obtained when applying a standard residual based adaptive strategy for the discretization of the homogenized problem (2.3) with piecewise linear FEM. Our indicator and data approximation are thus consistent with 
the classical theory. Furthermore, the lower and upper bounds stated below (see Theorems 19,20) also reduce to the classical bounds if using piecewise linear FEM for the numerical discretization of (2.3). We now prove the main theorem about a posteriori estimates. We follow [11].

Theorem 19 (Upper bound). Let $u_{0}, u^{H}$ be the solutions of problems (2.3) and (3.8), respectively. Then

$$
\left\|u_{0}-u^{H}\right\|_{H^{1}(\Omega)}^{2} \leq C\left(\eta_{H}(\Omega)^{2}+\xi_{H}(\Omega)^{2}\right),
$$

where $C$ only depends on the shape regularity constant, the coercivity and continuity bounds (2.2), the dimension $d$ and the Poincaré constant $C_{\Omega}$.

Proof. Using the representation formula (4.16), we obtain

$$
\begin{aligned}
B_{0}\left(e^{H}, e^{H}\right) & =\int_{\Omega} f_{H}\left(e^{H}-I_{H} e^{H}\right) d x+\int_{\Omega}\left(f-f_{H}\right)\left(e^{H}-I_{H} e^{H}\right) d x \\
& -\sum_{e \in \mathcal{E}} \int_{e} \llbracket \overline{a^{\varepsilon}(x) \nabla u_{K}^{h}} \rrbracket_{e}\left(e^{H}-I_{H} e^{H}\right) d s \\
& +\sum_{K \in \mathcal{T}_{H}} \int_{K}\left(a_{K}^{0}\left(x_{K}\right)-a^{0}(x)\right) \nabla u^{H} \nabla e^{H} d x,
\end{aligned}
$$

where $I_{H}$ is the Clément interpolation operator defined in Section 4.4. Notice that we used the identity $B\left(u^{H}, I_{H} e^{H}\right)=\sum_{K \in \mathcal{T}_{H}} \int_{K} f\left(I_{H} e^{H}\right) d x$ in the above equality. Defining $\phi^{H}:=e^{H}-I_{H} e^{H}$, using the interpolation estimates (4.17),(4.20) and the Cauchy-Schwarz inequality gives

$$
\begin{aligned}
& B_{0}\left(e^{H}, e^{H}\right) \leq C\left(\sum_{K \in \mathcal{T}_{H}} H_{K}\left\|f_{H}\right\|_{L^{2}(K)}\left\|\nabla e^{H}\right\|_{L^{2}(N(K))}\right. \\
& +\sum_{K \in \mathcal{T}_{H}} H_{K}\left\|\left(f-f_{H}\right)\right\|_{L^{2}(K)}\left\|\nabla e^{H}\right\|_{L^{2}(N(K))} \\
& +\sum_{e \in \mathcal{E}} H_{e}^{1 / 2}\left\|\int_{e} \llbracket \overline{\llbracket a^{\varepsilon}(x) \nabla u_{K}^{h}} \rrbracket_{e}\right\|_{L^{2}(e)}\left\|\nabla e^{H}\right\|_{L^{2}(N(e))} \\
& \left.+\sum_{K \in \mathcal{T}_{H}}\left\|\left(a_{K}^{0}\left(x_{K}\right)-a^{0}(x)\right) \nabla u^{H}\right\|_{L^{2}(K)}\left\|\nabla e^{H}\right\|_{L^{2}(K)}\right) .
\end{aligned}
$$

The coercivity of $B_{0}(\cdot, \cdot)$, the Poincaré inequality and the finite overlapping property of the neighborhoods $N(K)$

$$
\sum_{K \in \mathcal{T}_{H}}\left\|\nabla e^{H}\right\|_{L^{2}(N(K))} \leq C \sum_{K \in \mathcal{T}_{H}}\left\|\nabla e^{H}\right\|_{L^{2}(K)},
$$

where $C$ depends only on the shape regularity of the triangulation and the dimension $d$, allow to obtain the stated result. 
A priori and a posteriori analysis for numerical homogenization

As in the classical theory [34], we use bubble functions to derive a lower bound on the error. We recall the construction of such functions, belonging to a so-called bubble space. Let $\widetilde{\mathcal{T}}_{H}$ be a refinement of $\mathcal{T}_{H}$ such that every $K \in \mathcal{T}_{H}$ has an interior node $\tilde{x}_{K} \in K \backslash \partial K$ in $\widetilde{\mathcal{T}}_{H}$, and every edge $e$ of $\mathcal{T}_{H}$ not on the boundary $\partial \Omega$ has an interior node $x_{e} \in e \backslash \partial e$ in $\widetilde{\mathcal{T}}_{H}$. Based on the triangulation $\widetilde{\mathcal{T}}_{H}$ we define a piecewise linear FE space $S_{0}^{1}\left(\Omega, \widetilde{\mathcal{T}}_{H}\right) \supset S_{0}^{1}\left(\Omega, \mathcal{T}_{H}\right)$. Associated to $K \in \mathcal{T}_{H}$, we then consider a so-called bubble function $\psi_{K} \in S_{0}^{1}\left(\Omega, \widetilde{\mathcal{T}}_{H}\right)$ such that $0 \leq \psi_{K} \leq 1$, $\psi_{K}\left(\tilde{x}_{K}\right)=1$ and $\psi_{K} \equiv 0$ on $\Omega \backslash K$. For a finite dimensional (here piecewise constant) approximation $f_{H}$ of $f \in L^{2}(\Omega)$, we have,

$$
C\left\|f_{H}\right\|_{L^{2}(K)}^{2} \leq \int_{K} f_{H}\left(\psi_{K} f_{H}\right) d x
$$

where we used the equivalence of norms on a finite-dimensional space and where the constant $C$ is independent of $f_{H}$ and depends only on the shape regularity, the degree of the finite element space $S_{0}^{1}\left(\Omega, \widetilde{\mathcal{T}}_{H}\right)$ and the degree of the finite dimensional approximation $f_{H}$. For $K_{1}, K_{2} \in \mathcal{T}_{H}$ denote the common edge by $e=K_{1} \cap K_{2}$ and let $\psi_{e} \in S_{0}^{1}\left(\Omega, \widetilde{\mathcal{T}}_{H}\right)$ be a bubble function such that $\psi_{e}\left(x_{e}\right)=1, \psi_{e} \equiv 0$ on $\Omega \backslash\left(K_{1} \cup K_{2}\right)$. Using the equivalence of norms on a finite-dimensional space, we find that

$$
C H_{e}^{d-1} \leq \int_{e} \psi_{e} d s
$$

where $C$ is independent of $\psi_{e}$ and depends only on the shape regularity and the degree of the finite element space $S_{0}^{1}\left(\Omega, \widetilde{\mathcal{T}}_{H}\right)$. Using the CauchySchwarz inequality we also have $\left\|\psi_{e}\right\|_{L^{2}(K)} \leq C\left(H_{K}\right)^{d / 2}$ which yields, together with the inverse inequality (4.21),

$$
\left\|\nabla \psi_{e}\right\|_{L^{2}(K)} \leq C H_{K}^{-1}\left\|\psi_{e}\right\|_{L^{2}(K)} \leq C\left(H_{K}\right)^{\frac{d-2}{2}} .
$$

Theorem 20 (Lower bound). Let $u_{0}, u^{H}$ be the solutions of problems (2.3) and (3.8), respectively. Denote by $\omega_{K}$ the domain which consist of all elements sharing at least one side with $K$. Then

$$
\eta_{H}(K)^{2} \leq C\left(\left\|u_{0}-u^{H}\right\|_{H^{1}\left(\omega_{K}\right)}^{2}+\xi_{H}\left(\omega_{K}\right)^{2}\right),
$$

where $C$ only depends on the shape regularity constant, the coercivity and continuity bounds (2.2), the dimension $d$ and the Poincaré constant $C_{\Omega}$.

Proof. We start by estimating the so-called interior residual. Using the representation formula (4.16) with a test function given by $v:=\psi_{K} f_{H} \in$ 
$H_{0}^{1}(\Omega)$ gives

$$
\begin{aligned}
\int_{K} f_{H}\left(\psi_{K} f_{H}\right) d x= & B_{0}\left(e^{H}, \psi_{K} f_{H}\right)-\int_{K}\left(f-f_{H}\right) \psi_{K} f_{H} d x \\
& -\int_{K}\left(a_{K}^{0}\left(x_{K}\right)-a^{0}(x)\right) \nabla u^{H} \nabla\left(\psi_{K} f_{H}\right) d x .
\end{aligned}
$$

Using (6.6), $\left\|\left(\nabla \psi_{K} f_{H}\right)\right\|_{L^{2}(K)} \leq C H_{K}^{-1}\left\|\psi_{K} f_{H}\right\|_{L^{2}(K)}$ (the inverse inequality (4.21) for $\psi_{K} f_{H}$ ), the continuity of $B_{0}(\cdot, \cdot)$ and the CauchySchwarz inequality yields

$$
\begin{aligned}
H_{K}^{2}\left\|f_{H}\right\|_{L^{2}(K)}^{2} \leq & C\left(\left\|\nabla e^{H}\right\|_{L^{2}(K)}^{2}+H_{K}^{2}\left\|\left(f-f_{H}\right)\right\|_{L^{2}(K)}^{2}\right. \\
& \left.+\left\|\left(a_{K}^{0}\left(x_{K}\right)-a^{0}(x)\right) \nabla u^{H}\right\|_{L^{2}(K)}^{2}\right) .
\end{aligned}
$$

We now estimate the so-called jump residual. We use again the representation formula (4.16) with $v:=\psi_{e}$ and obtain

$$
\begin{aligned}
\int_{e} \llbracket \overline{a^{\varepsilon}(x) \nabla u_{K}^{h}} \rrbracket_{e} \psi_{e} d s & =\sum_{K_{1}, K_{2}}\left(\int_{K_{i}} f \psi_{e} d x-\int_{K_{i}} a^{0}(x) \nabla e^{H} \nabla \psi_{e} d x\right. \\
& \left.+\int_{K_{i}}\left(a_{K}^{0}\left(x_{K_{i}}\right)-a^{0}(x)\right) \nabla u^{H} \nabla \psi_{e} d x\right) .
\end{aligned}
$$

Using (6.7) gives $\int_{e} \llbracket \overline{a^{\varepsilon}(x) \nabla u_{K}^{h}} \rrbracket_{e} \psi_{e} d s \geq C H_{e}^{\frac{d-1}{2}}\left\|\llbracket \overline{\llbracket a^{\varepsilon}(x) \nabla u_{K}^{h}} \rrbracket_{e}\right\|_{L^{2}(e)}$ (notice that the multiscale flux is constant in $e$ ). The Cauchy-Schwarz inequality and the inequality (6.7) yields

$$
\begin{aligned}
H_{e}\left\|\overline{\llbracket \bar{a}^{\varepsilon}(x) \nabla u_{K}^{h}} \rrbracket_{e}\right\|_{L^{2}(e)}^{2} & \leq C \sum_{K_{1}, K_{2}}\left(H_{K_{i}}^{2}\left\|f_{H}\right\|_{L^{2}\left(K_{i}\right)}^{2}+\left\|\nabla e^{H}\right\|_{L^{2}\left(K_{i}\right)}^{2}\right. \\
& +H_{K_{i}}^{2}\left\|\left(f-f_{H}\right)\right\|_{L^{2}\left(K_{i}\right)}^{2} \\
& \left.+\left\|\left(a_{K}^{0}\left(x_{K_{i}}\right)-a^{0}(x)\right) \nabla u^{H}\right\|_{L^{2}\left(K_{i}\right)}^{2}\right),
\end{aligned}
$$

and using (6.9) we obtain

$$
\begin{aligned}
H_{e}\left\|\llbracket \overline{\llbracket a^{\varepsilon}(x) \nabla u_{K}^{h}} \rrbracket_{e}\right\|_{L^{2}(e)}^{2} & \leq C\left(\left\|\nabla e^{H}\right\|_{L^{2}\left(\omega_{e}\right)}^{2}+H_{\omega_{e}}^{2}\left\|\left(f-f_{H}\right)\right\|_{L^{2}\left(\omega_{e}\right)}^{2}\right. \\
& \left.+\left\|\left(a_{K}^{0}-a^{0}(x)\right) \nabla u^{H}\right\|_{L^{2}\left(\omega_{e}\right)}^{2}\right)
\end{aligned}
$$

where $H_{\omega_{e}}=\max _{i=1,2} H_{i}, \omega_{e}=K_{1} \cup K_{2}$. Adding $\frac{1}{2}$ of (6.11) for all $e \in \partial K$ to $(6.9)$ gives

$$
\eta_{H}(K)^{2} \leq C\left(\left\|u_{0}-u^{H}\right\|_{H^{1}\left(\omega_{K}\right)}^{2}+\xi_{H}\left(\omega_{K}\right)^{2}\right),
$$

and the proof is complete. 
A priori and a posteriori analysis for numerical homogenization 23

It remains to discuss the data approximation involved in $\xi_{H}$ (see (6.3)). The first term in (6.3) is standard and represents in general a higher order perturbation. For the second term we first have

$\left\|\left(a_{K}^{0}\left(x_{K}\right)-a^{0}(x)\right) \nabla u^{H}\right\|_{L^{2}(K)}^{2} \leq \sup _{x \in K}\left\|a_{K}^{0}\left(x_{K}\right)-a^{0}(x)\right\|_{F}^{2}\left\|\nabla u^{H}\right\|_{L^{2}(K)}^{2}$,

and we further have the following decomposition

$$
\begin{aligned}
\sup _{x \in K}\left\|a_{K}^{0}\left(x_{K}\right)-a^{0}(x)\right\|_{F}^{2} & \leq \underbrace{\sup _{x \in K}\left\|a^{0}(x)-a^{0}\left(x_{K}\right)\right\|_{F}^{2}}_{r_{M A C, K}}+\underbrace{\sup _{x \in K}\left\|a^{0}\left(x_{K}\right)-\bar{a}_{K}^{0}\left(x_{K}\right)\right\|_{F}^{2}}_{r_{M O D, K}} \\
& +\underbrace{\sup _{x \in K}\left\|\bar{a}_{K}^{0}\left(x_{K}\right)-a_{K}^{0}\left(x_{K}\right)\right\|_{F}^{2}}_{r_{M I C, K}},
\end{aligned}
$$

similarly as in Section 5. Estimates of the various terms are further detailed in the following theorem. As for the Theorem 17, all the constants are independent of $H, h$ and $\varepsilon$.

Theorem 21. For $K \in \mathcal{T}_{H}$, let $a_{K}^{0}(\cdot)$ be defined by (4.7). Assume that the homogenized tensor a $a^{0}$ (see (2.3)) satisfies (H1) (see Section (4.1)). Then,

$$
\sup _{x \in K}\left\|a_{K}^{0}\left(x_{K}\right)-a^{0}(x)\right\|_{F}^{2} \leq C_{1} H_{K}+r_{M I C, K}+r_{M O D, K} .
$$

If in addition to (Q1), (Q2) and (H1), (H2) hold with $q=1$ and $S^{1}\left(K_{\delta}, \mathcal{T}_{h}\right) \subset H_{0}^{1}\left(K_{\delta_{j}}\right)$, then

$$
\sup _{x \in K}\left\|a_{K}^{0}\left(x_{K}\right)-a^{0}(x)\right\|_{F}^{2} \leq C_{1} H_{K}+C_{2}\left(\frac{h}{\varepsilon}\right)^{2}+r_{M O D, K} .
$$

Alternatively, we also have (6.13) if (Q1), (Q2), (H1), (H2) (with $q=1)$ and (H3) hold, $\delta / \varepsilon \in \mathbb{N}$ and $S^{1}\left(K_{\delta}, \mathcal{T}_{h}\right) \subset W_{p e r}^{1}\left(K_{\delta_{j}}\right)$.

If in addition to (H1) and (H2) (with $q=1$ ), (H3) holds (see Section 4.3), then (6.13) hold with $r_{M O D, K}$ given by

$$
r_{M O D, K}:= \begin{cases}C_{3} \delta & \text { if } \delta / \varepsilon \in \mathbb{N}, S^{1}\left(K_{\delta}, \mathcal{T}_{h}\right) \subset W_{\text {per }}^{1}\left(K_{\delta}\right) ; \\ 0 & \text { if } \delta / \varepsilon \in \mathbb{N} \text { and } S^{1}\left(K_{\delta}, \mathcal{T}_{h}\right) \subset W_{p e r}^{1}\left(K_{\delta}\right), \text { the } \\ & \text { explicit decomposition } a^{\varepsilon}(x)=a(x, x / \varepsilon) \text { is } \\ & \text { available and collocation in the slow variable } \\ & \text { is used for the } F E-H M M ; \\ C_{4}\left(\delta+\frac{\varepsilon}{\delta}\right) & \text { if } \delta / \varepsilon \notin \mathbb{N} \text { and } S^{1}\left(K_{\delta}, \mathcal{T}_{h}\right) \subset H_{0}^{1}\left(K_{\delta}\right) .\end{cases}
$$


Proof. The proof of (6.12) follows directly from the Lipschitz continuity assumption of $a^{0}$. The proof of (6.13) follows from the Lemma 6. Finally, the proofs of the three estimates (6.14) follow from the Lemmata 7,8,9.

Micro refinement. As we refine adaptively the macro mesh $\mathcal{T}_{H}$ we have to appropriately refine the micro mesh in the sampling domains $K_{\delta}$ belonging to the macro elements marked for refinement. In view of Theorem 21 we see that

$$
\hat{h} \propto \sqrt{H_{K}}
$$

is an appropriate refinement strategy, where $\hat{h}=\frac{h}{\varepsilon}$.

Acknowledgments. The author thanks A. Nonnenmacher for a careful reading of the manuscript.

\section{References}

[1] A. Abdulle, On a-priori error analysis of Fully Discrete Heterogeneous Multiscale FEM, SIAM Multiscale Model. Simul., Vol. 4, No. 2 (2005), 447-459.

[2] A. Abdulle and C. Schwab, Heterogeneous Multiscale FEM for Diffusion Problem on Rough Surfaces, SIAM Multiscale Model. Simul., Vol. 3, No. 1 (2005), 195-220.

[3] A. Abdulle, Multiscale methods for advection-diffusion problems, Discrete Contin. Dyn. Syst. B, Suppl. Vol. (2005), 11-21.

[4] A. Abdulle, Analysis of a Heterogeneous Multiscale FEM for Problems in Elasticity, Math. Mod. Meth. Appl. Sci. (M3AS), Vol. 16, No. 2 (2006), 1-21.

[5] A. Abdulle, B. Engquist, Finite element heterogeneous multiscale methods with near optimal computational complexity, SIAM Multiscale Model. Simul. Vol. 6, No. 2 (2007), 1059-1084.

[6] A. Abdulle, Multiscale method based on discontinuous Galerkin methods for homogenization problems, C. R. Acad. Sci. Paris, Ser. I 346 (2008), 97-102.

[7] A. Abdulle, The finite element heterogeneous multiscale method: a computational strategy for multiscale PDEs, GAKUTO Int. Ser. Math. Sci. Appl. 31 (2009), 135-184.

[8] A. Abdulle, Discontinuous Galerkin finite element heterogeneous multiscale method for elliptic problems with multiple scales, preprint submitted for publication.

[9] A. Abdulle and A. Nonnenmacher, A short and versatile finite element multiscale code for homogenization problems, Comput. Methods Appl. Mech. Engrg. 198 (2009), 2839-2859. 
A priori and a posteriori analysis for numerical homogenization

[10] A. Abdulle, A. Nonnenmacher, A posteriori error analysis of the Heterogeneous Multiscale Method for homogenization problems, C. R. Acad. Sci. Paris, Ser. I. 247 (2009), 1081-1086.

[11] A. Abdulle, A. Nonnenmacher, Adaptive finite element heterogeneous multiscale method for homogenization problems, preprint submitted for publication.

[12] S. Agmon, Lectures on Elliptic Boundary Value Problems, Van Nostrand, Princeton, NJ, 1965.

[13] M. Ainsworth and J.T. Oden A Posteriori Error Estimation in Finite Element Analysis, John Wiley \& Sons, New York, 2000.

[14] G. Allaire, Homogenization and two-scale convergence, SIAM Journal on Mathematical Analysis, Vol. 23, No. 6 (1992), 1482-1518.

[15] T.J. Barth, T. Chan and R. Haimes, eds. Multiscale and multiresolution methods, theory and application, Springer, Lecture Notes in Computational Science and Engineering, Vol. 20, 2002.

[16] L. Bers, F. John, and M. Schechter, Partial Differential Equations, Lectures in Applied Mathematics, Proceedings of the Summer Seminar, Boulder, CO, 1957.

[17] P.G. Ciarlet and P.A. Raviart, The combined effect of curved boundaries and numerical integration in isoparametric finite element method, in A. K Aziz (Ed), Math. Foundation of the FEM with Applications to PDE, Academic Press, New York, NY, (1972), 409-474.

[18] P.G. Ciarlet, The Finite Element Method for Elliptic Problems, Society for Industrial and Applied Mathematics, 2002.

[19] D. Cioranescu and P. Donato, An introduction to Homogenization, Oxford University Press, 1999.

[20] P. Clément, Approximation by finite element functions using local regularization, Rev. Francaise Automat. Informat. Recherche Operationnelle Ser. Rouge Anal. Numer, Vol. 9 (1975), 77-84.

[21] E. De Giorgi and S. Spagnolo, Sulla convergenza degli integrali dell'energia per operatori ellittici del secondo ordine, Boll. Un. Mat. Ital., Vol. 4, No 8 (1973), 391-411.

[22] W. Dörfler, A convergent adaptive algorithm for Poisson's equation, SIAM J. Numer. Anal., Vol. 33 (1996), 1106-1124.

[23] W. E and B. Engquist, The Heterogeneous Multi-Scale Method, Commun. Math. Sci., Vol. 1, No. 1 (2003), 87-132.

[24] W. E, P. Ming and P. Zhang, Analysis of the heterogeneous multiscale method for elliptic homogenization problems, J. of AMS, V. 18, No 1 (2005), 121-156. 
[25] T. Y. Hou, Y. Efendiev, Multiscale Finite Element Methods, Surveys And Tutorials in the Applied Mathematical Sciences, Springer Verlag, 2009.

[26] V.V. Jikov, S.M. Kozlov and O.A. Oleinik, Homogenization of differential Operators and Integral Functionals, Springer-Verlag, Berlin, Heidelberg, 1994.

[27] O.A. Ladyzhenskaya, The Boundary Value Problems of Mathematical Physics, Applied Mathematical Sciences, 49, Springer-Verlag New York Inc., 1985.

[28] C. Miehe, J. Schröder and C. Bayreuther, On the homogenization analysis of composite materials based on discretized fluctuations on the micro-structure, Acta Mechanica, 135 (2002), 1-16.

[29] F. Murat and L. Tartar H-convergence, in "Topics in the mathematical modeling of composite materials", A. Cherkaev and R. Kohn Eds., Birkhäuser, Boston (1997), 21-43.

[30] G. Nguetseng, A general convergence result for a functional related to the theory of homogenization, SIAM J. Math. Anal., Vol. 20 (1989), $608-623$.

[31] J.T. Oden, S. Prudhomme, A. Romkes, and P. Bauman, Multiscale modeling of physical phenomena: Adaptive control of models, SIAM J. Sci. Comput., 28 (2006), 2359-2389.

[32] M. Ohlberger, A posteriori error estimates for the heterogeneous multiscale finite element method for elliptic homogenization problems, SIAM Multiscale Model. Simul. Vol. 4, No. 2 (2005), 88-114.

[33] R.M. Temam and A.M. Miranville, Mathematical Modeling in Continuum Mechanics, Cambridge University Press, 2d ed., 2005.

[34] R. Verfürth, A Review of a Posteriori Error Estimation and Adaptive Mesh-refinement Techniques, Wiley-Teubner, New York, 1996. 